\title{
Rigidez matinal en artritis reumatoidea: su relevancia en la evaluación de la enfermedad
}

\author{
Fernando Dal Pra \\ Médico de Staff, Sección Reumatología, Instituto de Rehabilitación Psicofísica.
}

En los últimos años, hemos visto importantes cambios en los criterios de clasificación, medidas de evaluación y pautas de tratamiento de la Artritis Reumatoidea (AR). Tanto los avances en el conocimiento fisiopatogénico de la enfermedad como los resultados a largo plazo de las cohortes de seguimiento longitudinales, han evidenciado la gravedad de la AR cuando ésta queda librada a su historia natural, viéndose esto reflejado en su potencial de progresión del daño articular y discapacidad, deterioro marcado de la calidad de vida y aumento de la mortalidad. Esa información pronóstica puso en evidencia la necesidad de un cambio de paradigma en el manejo de la enfermedad, apuntando principalmente a un diagnóstico temprano y a un tratamiento dirigido al objetivo, acompañado de un control estricto de la actividad inflamatoria. Este contexto exigió a su vez una revisión de las herramientas necesarias para diagnosticarla precozmente y evaluar adecuadamente la actividad, permitiendo los ajustes necesarios del tratamiento en búsqueda de remisión o baja actividad de la enfermedad.

En este marco de evaluación de la enfermedad encontramos, entre otras, a la valoración delarigidez matinal, cuya preponderancia en el contexto de otras medidas ha ido también cambiando a lo largo del tiempo si tomamos como ejemplo su exclusión de los criterios de clasificación ACR/EULAR 2010 o su ausencia en la mayoría de los índices compuestos de actividad o remisión. Sin embargo, recientemente ha habido quizás una revalorización de la misma, a través del mejor conocimiento de su patogenia basado en estudios actuales que la asocian con compromiso tenosinovial ${ }^{1}$, su consideración como criterio clínico para fases muy tempranas de la enfermedad (artritis clínicamente sospechosa) $)^{2}$ o como índice de respuesta a tratamiento en estudios aleatorizados y controlados ${ }^{3}$.

A pesar de esta revitalización de su importancia, aún no existe acuerdo en cuál es la mejor forma de evaluar la rigidez matinal ${ }^{4-5}$, estando al día de hoy en discusión si hacerlo mediante su intensidad, su duración o ambas. Aún más, se encuentra en desarrollo una forma más extensiva de evaluación de la rigidez bajo guías OMERACT, a través de cuestionarios multi-ítem, intentando determinar mejor su severidad e impactos físico y psicológico ${ }^{4}$. En tanto estas herramientas estén validadas y disponibles, continuamos valiéndonos de los datos que surgen de las formas clásicas de evaluación. En ese sentido, en el presente número de la Revista Argentina de Reumatología se presentan los resultados de un estudio multicéntrico en nuestro país, desarrollado por Barrios y cols., donde se evalúan diferentes maneras de valorar la rigidez matinal en AR (duración e intensidad, ya sea a través de la entrevista directa por el médico o en forma autorreportada mediante cuestionarios) y su correlación con índices de actividad y capacidad funcional. En ellos se observa que la rigidez matinal medida en minutos tiene una moderada a buena correlación con 
medidas de actividad y capacidad funcional. Así mismo, se observa que niveles de intensidad de la rigidez matinal mayores a 5,5 cm (en rango de 0 a 10) son muy específicos para predecir alta actividad inflamatoria de la enfermedad. Finalmente podemos concluir que, si bien la correlación de rigidez con medidas de actividad es aceptable, ésta dista de ser perfecta, lo que continúa justificando su uso como una medida independiente en la evaluación del paciente y el impacto en su capacidad funcional y calidad de vida.

\section{Bibliografía}

1. Kobayashi Y, Ikeda K, Nakamura T, Yamagata M, Nakazawa T, Tanaka S, et al. Severity and Diurnal Improvement of Morning Stiffness Independently Associate with Tenosynovitis in Patients with Rheumatoid Arthritis. PLoS One. 2016;11(11):e0166616.

2. van Steenbergen HW, Aletaha D, Beaart-van de Voorde LJJ, Brouwer E, Codreanu C, Combe B, et al. EULAR definition of arthralgia suspicious for progression to rheumatoid arthritis. Ann Rheum Dis. 2016; (Or 55):annrheumdis - 2016-209846.
3. Alten R, Holt R, Grahn A, Rice P, Kent J, Buttgereit F, et al. Morning stiffness response with delayed-release prednisone after ineffective course of immediaterelease prednisone ineffective course of immediaterelease prednisone. Scand J Rheumatol. Informa Healthcare; 2015;44(5):354-8.

4. Halls S, Sinnathurai P, Hewlett S, Mackie SL, March L, Bartlett SJ, et al. Stiffness Is the Cardinal Symptom of Inflammatory Musculoskeletal Diseases, Yet Still Variably Measured: Report from the OMERACT 2016 Stiffness Special Interest Group. J Rheumatol. 2016.

5. Yazici Y, Pincus T, Kautiainen H, Sokka T. Morning stiffness in patients with early rheumatoid arthritis is associated more strongly with functional disability than with joint swelling and erythrocyte sedimentation rate. J Rheumatol. 2004;31(9):1723-6. 